\title{
Mixed-order impulsive ordinary and fractional differential equations with initial conditions
}

\author{
Suphawat Asawasamrit ${ }^{1}$, Yasintorn Thadang ${ }^{1}$, Sotiris K. Ntouyas ${ }^{2,3}$ and Jessada Tariboon ${ }^{1 *}$ (D)
}

\section{"Correspondence:}

jessada.t@sci.kmutnb.ac.th

IIntelligent and Nonlinear Dynamic Innovations Research Center,

Department of Mathematics, Faculty of Applied Science, King Mongkut's University of Technology North Bangkok, Bangkok, Thailand

Full list of author information is available at the end of the article

\section{Springer}

\begin{abstract}
In this paper, using the idea of separated intervals in non-instantaneous impulsive equations, we initiate the study of initial value problems for mixed-order ordinary and fractional differential equations with instantaneous impulsive effects. Existence and uniqueness results are established via standard fixed point theorems. Examples illustrating the main results are also presented.
\end{abstract}

MSC: 26A33; 34B37

Keywords: Impulsive differential equations; Fractional impulsive differential equations; Instantaneous impulses; Non-instantaneous impulses

\section{Introduction and preliminaries}

Fractional differential equations have been shown to be very useful in the study of models of many phenomena in various fields of science and engineering, such as physics, chemistry, biology, signal and image processing, biophysics, blood flow phenomena, control theory, economics, aerodynamics, and fitting of experimental data. For examples and recent development of the topic, see [1-14] and the references cited therein.

Impulsive differential equations are used to describe many practical dynamical systems, including evolutionary processes characterized by abrupt changes of the state at certain instants. Such processes are naturally seen in biology, physics, engineering, and so forth. Due to their significance, many authors have established the solvability of impulsive differential equations. In the literature there are two popular types of impulses:

(i) Instantaneous impulses. The duration of these changes is relatively short compared to the overall duration of the whole process.

(ii) Non-instantaneous impulses. They are impulsive actions, starting abruptly at a fixed point and continuing on a finite time interval.

Differential equations with instantaneous impulses have been treated in several works, see, e.g., the monographs [15-17], papers [18-24], and the references therein.

A non-instantaneous impulsive differential equation was introduced by Hernandez and O'Regan [25] to describe a certain dynamic change of evolution processes in the pharmacotherapy. This kind of impulsive equations can be separated based on two major characteristics as differential equations on intervals $\left(s_{i}, t_{i+1}\right], i=0,1, \ldots, m$, and nonlinear equa-

(c) The Author(s) 2019. This article is distributed under the terms of the Creative Commons Attribution 4.0 International License (http://creativecommons.org/licenses/by/4.0/), which permits unrestricted use, distribution, and reproduction in any medium, provided you give appropriate credit to the original author(s) and the source, provide a link to the Creative Commons license, and indicate if changes were made. 
tions on $\left(t_{i}, s_{i}\right], i=1,2, \ldots, m$. For recent works, we refer the reader to the papers [26-31], books [32, 33], and the references therein.

In [34], it is pointed out that the necessary condition for non-instantaneous impulsive problems is having the explicit functions during impulsive intervals $\left(t_{i}, s_{i}\right], i=1,2,3, \ldots, m$. From that paper, we can conclude that the intervals of derivatives of the unknown function and those of the explicit given functions are alternately interchanged.

By using the separated intervals in non-instantaneous impulsive equations, we establish a new kind of mixed-order instantaneous impulsive differential equations which have one order derivative on $\left(s_{i}, t_{i+1}\right], i=0,1,2, \ldots, m$, and that of another order on $\left(t_{i}, s_{i}\right]$, $i=1,2,3, \ldots, m$. We study two initial value problems, one for mixed first and second ordinary derivatives and another for mixed fractional derivatives of orders $q$ and $p$ with $0<q \leq 1,1<p \leq 2$.

More precisely, in this paper, we study the existence and uniqueness of solutions for two new classes of instantaneous impulses of mixed-order ordinary differential equations, as well as, fractional differential equations with initial conditions. The first problem for mixed-order ordinary impulsive differential equations is presented by

$$
\left\{\begin{array}{l}
x^{\prime \prime}(t)=f(t, x(t)), \quad t \in\left(s_{i}, t_{i+1}\right], i=0,1,2, \ldots, m, \\
x^{\prime}(t)=g(t, x(t)), \quad t \in\left(t_{i}, s_{i}\right], i=1,2,3, \ldots, m, \\
x\left(s_{i}^{+}\right)=\alpha_{i} x\left(s_{i}^{-}\right), \quad x^{\prime}\left(s_{i}^{+}\right)=\beta_{i} x^{\prime}\left(s_{i}^{-}\right), \\
x\left(t_{i}^{+}\right)=\gamma_{i} x\left(t_{i}^{-}\right), \quad x(0)=\alpha_{0}, \quad x^{\prime}(0)=\beta_{0},
\end{array}\right.
$$

while the second for fractional mixed-order is given by

$$
\left\{\begin{array}{l}
s_{i}^{+} D^{p} x(t)=f(t, x(t)), \quad t \in\left(s_{i}, t_{i+1}\right], i=0,1,2, \ldots, m, \\
t_{i}^{+} D^{q} x(t)=g(t, x(t)), \quad t \in\left(t_{i}, s_{i}\right], i=1,2,3, \ldots, m, \\
x\left(s_{i}^{+}\right)=\alpha_{i} x\left(s_{i}^{-}\right), \quad x^{\prime}\left(s_{i}^{+}\right)=\beta_{i} x^{\prime}\left(s_{i}^{-}\right), \\
x\left(t_{i}^{+}\right)=\gamma_{i} x\left(t_{i}^{-}\right), \quad x(0)=\alpha_{0}, \quad x^{\prime}(0)=\beta_{0},
\end{array}\right.
$$

where we have nonlinear functions $f: J \times \mathbb{R} \rightarrow \mathbb{R}, g: J^{*} \times \mathbb{R} \rightarrow \mathbb{R} ; J=\bigcup_{i=0}^{m}\left(s_{i}, t_{i+1}\right], J^{*}=$ $\bigcup_{i=1}^{m}\left(t_{i}, s_{i}\right], J \cup J^{*} \cup\{0\}=[0, T], T=t_{m+1}$, constants $\alpha_{i}, \beta_{i}$, for $i=0,1, \ldots, m$, and $\gamma_{i}, i=$ $1,2, \ldots, m$, are given, and $s_{i}^{+} D^{p}, t_{i}^{+} D^{q}$ denote the Caputo fractional derivatives of orders $p$ and $q, 1<p \leq 2,0<q \leq 1$, starting at the points $s_{i}^{+}, i=0,1, \ldots, m$, and $t_{i}^{+}, i=1,2, \ldots, m$.

To the best of the authors' knowledge, problems (1.1) and (1.2) are new mixed-order impulsive ordinary and fractional differential equations, respectively. The system of integer order derivatives in Eq. (1.1) can be used to explain a mixture of growth, decay, and transient phenomena, while Eq. (1.2) gives some details for description of memory and hereditary properties of various materials and processes with impulses. Observe that problem (1.2) contains the interchanging fractional orders, for $1<p \leq 2$ and $0<q \leq 1$, of Caputo type, which has the property of the derivative constant equal to zero and can be suitably used to establish impulsive systems.

Note that problem (1.2) is well-defined in the sense of Caputo fractional derivative of impulsive problem as

$$
{ }_{\phi} D^{\theta} x(t)=\frac{1}{\Gamma(n-\theta)} \int_{\phi}^{t}(t-r)^{n-\theta-1} x^{(n)}(r) d r,
$$


where $n-1<\theta<n, n=[\theta]+1$, and $[\theta]$ denotes the integer part of the real number $\theta$. Indeed, either $t \in J, \phi=s_{i}^{+}$, or $t \in J^{*}, \phi=t_{i}^{+}$, shows that Eq. (1.3) does not contain impulse effects of unknown variable $x$ on the interval of integration. Therefore $x^{(n)}(r)$ in Eq. (1.3) exists for all $r \in(\phi, t)$. Some details of fractional calculus for impulsive problems, we refer the reader to [18]. As is customary, the Riemann-Liouville fractional integral of $x(t)$ is defined by

$$
{ }_{\phi} I^{\theta} x(t)=\frac{1}{\Gamma(\theta)} \int_{\phi}^{t} \frac{x(r)}{(t-r)^{1-\theta}} d r, \quad \theta>0
$$

provided the integral exists. In addition, we also use the following formula to establish our results:

$$
{ }_{\phi} I^{\theta}\left({ }_{\phi} D^{\theta} x\right)(t)=x(t)+c_{0}+c_{1}(t-\phi)+\cdots+c_{n-1}(t-\phi)^{n-1},
$$

for some $c_{i} \in \mathbb{R}, i=0,1,2, \ldots, n-1(n=[\theta]+1)$.

\section{Main results}

To prove the existence and uniqueness results for problems (1.1) and (1.2), we have to define the structure of the sets of piecewise functions. Now, let us define two increasing sequences of points $\left\{t_{i}\right\}_{i=1}^{m+1}$ and $\left\{s_{i}\right\}_{i=0}^{m}$ by

$$
0=s_{0}<t_{1} \leq s_{1}<t_{2} \leq s_{2}<t_{3} \leq \cdots<t_{m} \leq s_{m}<t_{m+1}=T .
$$

Moreover, we define $U=J \cup J^{*} \cup\{0\}$, as well as the sets $P C(J, \mathbb{R})=\{x: J \rightarrow \mathbb{R} ; x(t)$ is continuous on $J$ and $x\left(s_{i}^{+}\right), x^{\prime}\left(s_{i}^{+}\right)$exist for $\left.i=0,1, \ldots, m\right\}$ and $P C\left(J^{*}, \mathbb{R}\right)=\left\{x: J^{*} \rightarrow \mathbb{R} ; x(t)\right.$ is continuous on $J^{*}$ and $x\left(t_{i}^{+}\right)$exist for $\left.i=1,2, \ldots, m\right\}$. In addition, we also define $P C_{u}^{p}(J, \mathbb{R})=$ $\left\{x \in P C(J, \mathbb{R}):{ }_{u} D^{p} x(t)\right.$ is continuous everywhere for $t \in J$ for $u \in\left\{s_{i}^{+}: i=0,1, \ldots, m\right\}, 1<$ $p \leq 2\}, P C_{v}^{q}\left(J^{*}, \mathbb{R}\right)=\left\{x \in P C\left(J^{*}, \mathbb{R}\right):{ }_{\nu} D^{q} x(t)\right.$ is continuous everywhere for $t \in J^{*}, v \in\left\{t_{i}^{+}\right.$: $i=1,2, \ldots, m\}, 0<q \leq 1\}$, and $P C^{p, q}(U, \mathbb{R})=P C_{u}^{p}(J, \mathbb{R}) \cup P C_{v}^{q}\left(J^{*}, \mathbb{R}\right)$. Further, the space $P C^{p, q}(U, \mathbb{R})$ is a Banach space endowed with the norm defined by $\|x\|=\sup _{t \in U}|x(t)|$.

Let us define several constants as follows:

$$
\begin{aligned}
\Lambda_{1}^{(p)}= & \frac{\left(T-s_{m}\right)^{p}}{\Gamma(p+1)}+\sum_{j=1}^{m}\left(\prod_{j}^{m}\left|\alpha_{j}\right|\left|\gamma_{j}\right| \frac{\left(t_{j}-s_{j-1}\right)^{p}}{\Gamma(p+1)}\right), \\
\Lambda_{2}^{(q)}= & \sum_{j=1}^{m}\left(\prod_{j}^{m}\left|\alpha_{j}\right| \prod_{j+1}^{m}\left|\gamma_{j+1}\right| \frac{\left(s_{j}-t_{j}\right)^{q}}{\Gamma(q+1)}\right)+\sum_{j=1}^{m}\left(\prod_{j}^{m}\left|\alpha_{j}\right|\left|\gamma_{j}\right|\right)\left|\beta_{j-1}\right| t_{j}+\left|\beta_{m}\right| T, \\
\Lambda_{3}^{(p)}= & \left|\gamma_{m}\right| \sum_{j=1}^{m}\left[\left(\prod_{j}^{m-1}\left|\alpha_{j}\right|\left|\gamma_{j}\right|\right) \frac{\left(t_{j}-s_{j-1}\right)^{p}}{\Gamma(p+1)}\right], \\
\Lambda_{4}^{(q)}= & \left|\gamma_{m}\right|\left[\sum_{j=1}^{m-1}\left(\prod_{j}^{m-1}\left|\alpha_{j}\right|\right)\left(\prod_{j+1}^{m-1}\left|\gamma_{j+1}\right|\right) \frac{\left(s_{j}-t_{j}\right)^{q}}{\Gamma(q+1)}+\sum_{j=1}^{m}\left(\prod_{j}^{m-1}\left|\alpha_{j}\right|\left|\gamma_{j}\right|\right)\left|\beta_{j-1}\right| t_{j}\right] \\
& +\frac{\left(s_{m}-t_{m}\right)^{q}}{\Gamma(q+1)}
\end{aligned}
$$




$$
\Phi_{1}=\left|\alpha_{0}\right| \prod_{j=1}^{m}\left|\alpha_{j}\right|\left|\gamma_{j}\right|, \quad \Phi_{2}=\left|\gamma_{m}\right|\left|\alpha_{0}\right|\left(\prod_{j=1}^{m-1}\left|\alpha_{j}\right|\left|\gamma_{j}\right|\right) .
$$

Observe that $P C^{2,1}(U, \mathbb{R})$ is used to study problem (1.1). In addition, the constants $\Lambda_{1}^{(2)}$, $\Lambda_{2}^{(1)}, \Lambda_{3}^{(2)}$, and $\Lambda_{4}^{(1)}$ appear in the next subsection.

\subsection{Mixed-order impulsive ordinary differential equations with initial conditions}

In this subsection, we establish a mixture of first and second order ordinary impulsive differential equations by transforming the initial value problem to an integral equation. Using mathematical induction, the following theorem for a linear problem is proved.

Theorem 2.1 Let $\alpha_{j}, \beta_{j}$ be given constants for $j=0,1,2, \ldots, m$, and $\gamma_{i} \in \mathbb{R}$ for $i=$ $1,2,3, \ldots, m$. Consider the functions $y: J \rightarrow \mathbb{R}$ and $z: J^{*} \rightarrow \mathbb{R}$, with $z\left(s_{0}\right)=1$. The impulsive problem

$$
\left\{\begin{array}{l}
x^{\prime \prime}(t)=y(t), \quad t \in\left(s_{i}, t_{i+1}\right], i=0,1,2, \ldots, m, \\
x^{\prime}(t)=z(t), \quad t \in\left(t_{i}, s_{i}\right], i=1,2,3, \ldots, m, \\
x\left(s_{i}^{+}\right)=\alpha_{i} x\left(s_{i}^{-}\right), \quad x^{\prime}\left(s_{i}^{+}\right)=\beta_{i} x^{\prime}\left(s_{i}^{-}\right), \\
x\left(t_{i}^{+}\right)=\gamma_{i} x\left(t_{i}^{-}\right), \quad x(0)=\alpha_{0}, \quad x^{\prime}(0)=\beta_{0},
\end{array}\right.
$$

can be rewritten as a linear integral equation in the following form:

$$
\begin{aligned}
x(t)= & \alpha_{0}\left(\prod_{j=1}^{i} \alpha_{j} \gamma_{j}\right)+\sum_{j=1}^{i}\left[\left(\prod_{j}^{i} \alpha_{j}\right)\left(\prod_{j+1}^{i} \gamma_{j+1}\right) \int_{t_{j}}^{s_{j}} z(r) d r\right] \\
& +\sum_{j=1}^{i}\left[\left(\prod_{j}^{i} \alpha_{j} \gamma_{j}\right) \int_{s_{j-1}}^{t_{j}}\left(t_{j}-r\right) y(r) d r\right]+\sum_{j=1}^{i}\left(\prod_{j}^{i} \alpha_{j} \gamma_{j}\right) \beta_{j-1} z\left(s_{j-1}\right) t_{j} \\
& +\beta_{i} z\left(s_{i}\right) t+\int_{s_{i}}^{t}(t-r) y(r) d r, \quad t \in J,
\end{aligned}
$$

and

$$
\begin{aligned}
x(t)= & \alpha_{0} \gamma_{i}\left(\prod_{j=1}^{i-1} \alpha_{j} \gamma_{j}\right)+\gamma_{i} \sum_{j=1}^{i-1}\left[\left(\prod_{j}^{i-1} \alpha_{j}\right)\left(\prod_{j+1}^{i-1} \gamma_{j+1}\right) \int_{t_{j}}^{s_{j}} z(r) d r\right] \\
& +\gamma_{i}\left[\sum_{j=1}^{i}\left(\prod_{j}^{i-1} \alpha_{j} \gamma_{j}\right) \int_{s_{j-1}}^{t_{j}}\left(t_{j}-r\right) y(r) d r\right]+\gamma_{i} \sum_{j=1}^{i}\left(\prod_{j}^{i-1} \alpha_{j} \gamma_{j}\right) \beta_{j-1} z\left(s_{j-1}\right) t_{j} \\
& +\int_{t_{i}}^{t} z(r) d r, \quad t \in J^{*} .
\end{aligned}
$$

Proof For $t \in\left(s_{0}, t_{1}\right]$, the first ordinary differential equation of second order in Eq. (2.1) can be written as

$$
x(t)=\alpha_{0}+\beta_{0} t+\int_{s_{0}}^{t}(t-r) y(r) d r
$$


with $\prod_{1}^{0}(\cdot)=1, \sum_{1}^{0}(\cdot)=0$ and $z\left(s_{0}\right)=1$. Then Eq. (2.2) holds for $i=0$. Now, for $t \in\left(t_{1}, s_{1}\right]$, by integrating the second equation of Eq. (2.1) from $t_{1}$ to $t$, when $t \in\left(t_{1}, s_{1}\right]$, we have

$$
x(t)=x\left(t_{1}^{+}\right)+\int_{t_{1}}^{t} z(r) d r .
$$

Applying the condition $x\left(t_{1}^{+}\right)=\gamma_{1} x\left(t_{1}^{-}\right)$, we obtain

$$
x(t)=\gamma_{1} \alpha_{0}+\gamma_{1} t_{1} \beta_{0}+\gamma_{1} \int_{s_{0}}^{t_{1}}\left(t_{1}-r\right) y(r) d r+\int_{t_{1}}^{t} z(r) d r,
$$

which implies that Eq. (2.3) is true for $i=1$. In the next step, we assume that Eq. (2.2) holds for $t \in\left(s_{i}, t_{i+1}\right]$. By mathematical induction, we will show that Eq. (2.3) is fulfilled for $t \in\left(t_{i+1}, s_{i+1}\right]$. Indeed, for $t \in\left(t_{i+1}, s_{i+1}\right]$, we get

$$
x(t)=x\left(t_{i+1}^{+}\right)+\int_{t_{i+1}}^{t} z(r) d r
$$

From $x\left(t_{i+1}^{+}\right)=\gamma_{i+1} x\left(t_{i+1}^{-}\right)$and Eq. (2.2), Eq. (2.4) can be expressed as

$$
\begin{aligned}
x(t)= & \alpha_{0} \gamma_{i+1}\left(\prod_{j=1}^{i} \alpha_{j} \gamma_{j}\right)+\gamma_{i+1} \sum_{j=1}^{i}\left[\left(\prod_{j}^{i} \alpha_{j}\right)\left(\prod_{j+1}^{i} \gamma_{j+1}\right) \int_{t_{j}}^{s_{j}} z(r) d r\right] \\
& +\gamma_{i+1}\left[\sum_{j=1}^{i+1}\left(\prod_{j}^{i} \alpha_{j} \gamma_{j}\right) \int_{s_{j-1}}^{t_{j}}\left(t_{j}-r\right) y(r) d r\right] \\
& +\gamma_{i+1} \sum_{j=1}^{i+1}\left(\prod_{j}^{i} \alpha_{j} \gamma_{j}\right) \beta_{j-1} z\left(s_{j-1}\right) t_{j}+\int_{t_{i+1}}^{t} z(r) d r .
\end{aligned}
$$

Thus Eq. (2.3) is satisfied for $t \in\left(t_{i+1}, s_{i+1}\right]$.

Finally, we suppose that Eq. (2.3) is true for $\left(t_{i}, s_{i}\right]$ and then we will prove that Eq. (2.2) is true for $\left(s_{i}, t_{i+1}\right]$. From the first equation of Eq. (2.1), we obtain

$$
x(t)=x\left(s_{i}^{+}\right)+x^{\prime}\left(s_{i}^{+}\right) t+\int_{s_{i}}^{t}(t-r) y(r) d r .
$$

Using Eq. (2.3) and conditions $x\left(s_{i}^{+}\right)=\alpha_{i} x\left(s_{i}^{-}\right), x^{\prime}\left(s_{i}^{+}\right)=\beta_{i} x^{\prime}\left(s_{i}^{-}\right)$, we have

$$
\begin{aligned}
x(t)= & \alpha_{i}\left\{\alpha_{0} \gamma_{i}\left(\prod_{j=1}^{i-1} \alpha_{j} \gamma_{j}\right)+\gamma_{i} \sum_{j=1}^{i-1}\left[\left(\prod_{j}^{i-1} \alpha_{j}\right)\left(\prod_{j+1}^{i-1} \gamma_{j+1}\right) \int_{t_{j}}^{s_{j}} z(r) d r\right]\right. \\
& +\gamma_{i}\left[\sum_{j=1}^{i}\left(\prod_{j}^{i-1} \alpha_{j} \gamma_{j}\right) \int_{s_{j-1}}^{t_{j}}\left(t_{j}-r\right) y(r) d r\right]+\gamma_{i} \sum_{j=1}^{i}\left(\prod_{j}^{i-1} \alpha_{j} \gamma_{j}\right) \beta_{j-1} z\left(s_{j-1}\right) t_{j} \\
& \left.+\int_{t_{i}}^{s_{i}} z(r) d r\right\}+\beta_{i} z\left(s_{i}\right) t+\int_{s_{i}}^{t}(t-r) y(r) d r .
\end{aligned}
$$

Therefore Eq. (2.2) is valid on $\left(s_{i}, t_{i+1}\right]$. The converse follows by direct computation. This completes the proof. 
Next, in view of Theorem 2.1, replacing linear functions $y(t), z(t)$ by nonlinear functions $f(t, x), g(t, x)$, respectively, we define the operator $\mathcal{A}: P C^{2,1}(U, \mathbb{R}) \rightarrow P C^{2,1}(U, \mathbb{R})$ by

$$
\mathcal{A} x(t)=\left\{\begin{aligned}
\alpha_{0}\left(\prod_{j=1}^{i} \alpha_{j} \gamma_{j}\right)+\sum_{j=1}^{i}\left[\left(\prod_{j}^{i} \alpha_{j}\right)\left(\prod_{j+1}^{i} \gamma_{j+1}\right) \int_{t_{j}}^{s_{j}} g(r, x(r)) d r\right] \\
\quad+\sum_{j=1}^{i}\left[\left(\prod_{j}^{i} \alpha_{j} \gamma_{j}\right) \int_{s_{j-1}}^{t_{j}}\left(t_{j}-r\right) f(r, x(r)) d r\right] \\
\quad+\sum_{j=1}^{i}\left(\prod_{j}^{i} \alpha_{j} \gamma_{j}\right) \beta_{j-1} g\left(s_{j-1}, x\left(s_{j-1}\right)\right) t_{j} \\
\quad+\beta_{i} g\left(s_{i}, x\left(s_{i}\right)\right) t+\int_{s_{i}}^{t}(t-r) f(r, x(r)) d r, \quad t \in J, \\
\alpha_{0} \gamma_{i}\left(\prod_{j=1}^{i-1} \alpha_{j} \gamma_{j}\right)+\gamma_{i} \sum_{j=1}^{i-1}\left[\left(\prod_{j}^{i-1} \alpha_{j}\right)\left(\prod_{j+1}^{i-1} \gamma_{j+1}\right) \int_{t_{j}}^{s_{j}} g(r, x(r)) d r\right] \\
\quad+\gamma_{i}\left[\sum_{j=1}^{i}\left(\prod_{j}^{i-1} \alpha_{j} \gamma_{j}\right) \int_{s_{j-1}}^{t_{j}}\left(t_{j}-r\right) f(r, x(r)) d r\right] \\
\quad+\gamma_{i} \sum_{j=1}^{i}\left(\prod_{j}^{i-1} \alpha_{j} \gamma_{j}\right) \beta_{j-1} g\left(s_{j-1}, x\left(s_{j-1}\right)\right) t_{j} \\
\quad+\int_{t_{i}}^{t} g(r, x(r)) d r, \quad t \in J^{*} .
\end{aligned}\right.
$$

Then problem (1.1) is transformed to the operator equation $x=\mathcal{A} x$, which is the fixed point problem. The existence of a unique solution is proved by using the Banach contraction mapping principle.

Theorem 2.2 Assume that the functions $f: J \times \mathbb{R} \rightarrow \mathbb{R}$ and $g: J^{*} \times \mathbb{R} \rightarrow \mathbb{R}$ with $g(0, \cdot)=1$ satisfy:

$\left(\mathrm{H}_{1}\right)$ There exist positive constants $L_{1}, L_{2}$ such that

$$
\left|f\left(t_{1}, x\right)-f\left(t_{1}, y\right)\right| \leq L_{1}|x-y| \text { and }\left|g\left(t_{2}, x\right)-g\left(t_{2}, y\right)\right| \leq L_{2}|x-y|
$$

for all $t_{1} \in J, t_{2} \in J^{*}$ and $x, y \in \mathbb{R}$.

If $\Omega_{1}=\max \left\{L_{1} \Lambda_{1}^{(2)}+L_{2} \Lambda_{2}^{(1)}, L_{1} \Lambda_{3}^{(2)}+L_{2} \Lambda_{4}^{(1)}\right\}<1$, then problem (1.1) has a unique solution on $U$ such that $\|x\| \leq r^{*}$ with $r^{*}=\max \left\{r_{1}, r_{2}\right\}$,

$$
r_{1}=\frac{M_{1} \Lambda_{1}^{(2)}+M_{2} \Lambda_{2}^{(1)}+\Phi_{1}}{1-\left(L_{1} \Lambda_{1}^{(2)}+L_{2} \Lambda_{2}^{(1)}\right)}, \quad r_{2}=\frac{M_{1} \Lambda_{3}^{(2)}+M_{2} \Lambda_{4}^{(1)}+\Phi_{2}}{1-\left(L_{1} \Lambda_{3}^{(2)}+L_{2} \Lambda_{4}^{(1)}\right)},
$$

and $M_{1}=\sup _{t \in J}\{f(t, 0)\}, M_{2}=\sup _{t \in J^{*}}\{g(t, 0)\}$.

Proof Let us consider a ball $B_{r^{*}}=\left\{x \in P C^{2,1}:\|x\| \leq r^{*}\right\}$. We will show that the operator $\mathcal{A}$ satisfies $\mathcal{A} B_{r^{*}} \subset B_{r^{*}}$. For $t \in\left(s_{i}, t_{i+1}\right], i=0,1,2, \ldots, m$ and by using $|\phi(r, x(r))| \leq \mid \phi(r, x(r))-$ $\phi(r, 0)|+| \phi(r, 0) \mid, \phi \in\{f, g\}$, we find that

$$
\begin{aligned}
|\mathcal{A} x(t)| \leq & \left|\alpha_{0}\right|\left(\prod_{j=1}^{m}\left|\alpha_{j}\right|\left|\gamma_{j}\right|\right)+\sum_{j=1}^{m}\left[\left(\prod_{j}^{m}\left|\alpha_{j}\right|\right)\left(\prod_{j+1}^{m}\left|\gamma_{j+1}\right|\right) \int_{t_{j}}^{s_{j}}|g(r, x(r))| d r\right] \\
& +\sum_{j=1}^{m}\left[\left(\prod_{j}^{m}\left|\alpha_{j}\right|\left|\gamma_{j}\right|\right) \int_{s_{j-1}}^{t_{j}}\left(t_{j}-r\right)|f(r, x(r))| d r\right] \\
& +\sum_{j=1}^{m}\left(\prod_{j}^{m}\left|\alpha_{j}\right|\left|\gamma_{j}\right|\right)\left|\beta_{j-1}\right|\left|g\left(s_{j-1}, x\left(s_{j-1}\right)\right)\right| t_{j} \\
& +\left|\beta_{m}\right|\left|g\left(s_{m}, x\left(s_{m}\right)\right)\right| T+\int_{s_{m}}^{T}(T-r)|f(r, x(r))| d r
\end{aligned}
$$




$$
\begin{aligned}
\leq & \left|\alpha_{0}\right|\left(\prod_{j=1}^{m}\left|\alpha_{j}\right|\left|\gamma_{j}\right|\right) \\
& +\sum_{j=1}^{m}\left[\left(\prod_{j}^{m}\left|\alpha_{j}\right|\right)\left(\prod_{j+1}^{m}\left|\gamma_{j+1}\right|\right) \int_{t_{j}}^{s_{j}}(|g(r, x(r))-g(r, 0)|+|g(r, 0)|) d r\right] \\
& +\sum_{j=1}^{m}\left[\left(\prod_{j}^{m}\left|\alpha_{j}\right|\left|\gamma_{j}\right|\right) \int_{s_{j-1}}^{t_{j}}\left(t_{j}-r\right)(|f(r, x(r))-f(r, 0)|+|f(r, 0)|) d r\right] \\
& +\sum_{j=1}^{m}\left(\prod_{j}^{m}\left|\alpha_{j}\right|\left|\gamma_{j}\right|\right)\left|\beta_{j-1}\right|\left(\left|g\left(s_{j-1}, x\left(s_{j-1}\right)\right)-g(r, 0)\right|+|g(r, 0)|\right) t_{j} \\
& +\left|\beta_{m}\right|\left(\left|g\left(s_{m}, x\left(s_{m}\right)\right)-g\left(s_{m}, 0\right)\right|+\left|g\left(s_{m}, 0\right)\right|\right) T \\
& +\int_{s_{m}}^{T}(T-r)(|f(r, x(r))-f(r, 0)|+|f(r, 0)|) d r \\
\leq & \left(L_{1} r_{1}+M_{1}\right)\left[\frac{\left(T-s_{m}\right)^{2}}{2}+\sum_{j=1}^{m}\left(\prod_{j}^{m}\left|\alpha_{j}\right|\left|\gamma_{j}\right| \frac{\left(t_{j}-s_{j-1}\right)^{2}}{2}\right)\right] \\
& +\left(L_{2} r_{1}+M_{2}\right)\left[\sum_{j=1}^{m}\left(\prod_{j}^{m}\left|\alpha_{j}\right| \prod_{j+1}^{m}\left|\gamma_{j+1}\right|\left(s_{j}-t_{j}\right)\right)\right. \\
& \left.+\sum_{j=1}^{m}\left(\prod_{j}^{m}\left|\alpha_{j}\right|\left|\gamma_{j}\right|\right)\left|\beta_{j-1}\right| t_{j}+\left|\beta_{m}\right| T\right]+\left|\alpha_{0}\right| \prod_{j=1}^{m}\left|\alpha_{j}\right|\left|\gamma_{j}\right| \\
= & \left(L_{1} r_{1}+M_{1}\right) \Lambda_{1}^{(2)}+\left(L_{2} r_{1}+M_{2}\right) \Lambda_{2}^{(1)}+\Phi_{1} \\
\leq & r_{1} .
\end{aligned}
$$

For $t \in\left(t_{i}, s_{i}\right], i=1,2,3, \ldots, m$, we obtain

$$
\begin{aligned}
& |\mathcal{A} x(t)| \\
& \leq\left|\gamma_{m}\right|\left|\alpha_{0}\right|\left(\prod_{j=1}^{m-1}\left|\alpha_{j}\right|\left|\gamma_{j}\right|\right)+\left|\gamma_{m}\right| \sum_{j=1}^{m-1}\left[\left(\prod_{j}^{m-1}\left|\alpha_{j}\right|\right)\left(\prod_{j+1}^{m-1}\left|\gamma_{j+1}\right|\right) \int_{t_{j}}^{s_{j}}|g(r, x(r))| d r\right] \\
& +\left|\gamma_{m}\right| \sum_{j=1}^{m}\left[\left(\prod_{j}^{m-1}\left|\alpha_{j}\right|\left|\gamma_{j}\right|\right) \int_{s_{j-1}}^{t_{j}}\left(t_{j}-r\right)|f(r, x(r))| d r\right] \\
& +\left|\gamma_{m}\right| \sum_{j=1}^{m}\left(\prod_{j}^{m-1}\left|\alpha_{j}\right|\left|\gamma_{j}\right|\right)\left|\beta_{j-1}\right|\left|g\left(s_{j-1}, x\left(s_{j-1}\right)\right)\right| t_{j}+\int_{t_{m}}^{s_{m}}|g(r, x(r))| d r d r \\
& \leq\left|\gamma_{m}\right|\left|\alpha_{0}\right|\left(\prod_{j=1}^{m-1}\left|\alpha_{j}\right|\left|\gamma_{j}\right|\right) \\
& \quad+\left|\gamma_{m}\right| \sum_{j=1}^{m-1}\left[\left(\prod_{j}^{m-1}\left|\alpha_{j}\right|\right)\left(\prod_{j+1}^{m-1}\left|\gamma_{j+1}\right|\right) \int_{t_{j}}^{s_{j}}(|g(r, x(r))-g(r, 0)|+|g(r, 0)|) d r\right] \\
& \quad+\left|\gamma_{m}\right| \sum_{j=1}^{m}\left[\left(\prod_{j}^{m-1}\left|\alpha_{j}\right|\left|\gamma_{j}\right|\right) \int_{s_{j-1}}^{t_{j}}\left(t_{j}-r\right)(|f(r, x(r))-f(r, 0)|+|f(r, 0)|) d r\right]
\end{aligned}
$$




$$
\begin{aligned}
& +\left|\gamma_{m}\right| \sum_{j=1}^{m}\left(\prod_{j}^{m-1}\left|\alpha_{j}\right|\left|\gamma_{j}\right|\right)\left|\beta_{j-1}\right|\left(\left|g\left(s_{j-1}, x\left(s_{j-1}\right)\right)-g\left(s_{j-1}, 0\right)\right|+\left|g\left(s_{j-1}, 0\right)\right|\right) t_{j} \\
& +\int_{t_{m}}^{s_{m}}(|g(r, x(r))-g(r, 0)|+|g(r, 0)|) d r \\
\leq & \left|\gamma_{m}\right|\left|\alpha_{0}\right|\left(\prod_{j=1}^{m-1}\left|\alpha_{j}\right|\left|\gamma_{j}\right|\right) \\
& +\left(L_{2} r_{2}+M_{2}\right)\left|\gamma_{m}\right| \sum_{j=1}^{m-1}\left[\left(\prod_{j}^{m-1}\left|\alpha_{j}\right|\right)\left(\prod_{j+1}^{m-1}\left|\gamma_{j+1}\right|\right)\left(s_{j}-t_{j}\right)\right] \\
& +\left(L_{1} r_{2}+M_{1}\right)\left|\gamma_{m}\right| \sum_{j=1}^{m}\left[\left(\prod_{j}^{m-1}\left|\alpha_{j}\right|\left|\gamma_{j}\right|\right) \frac{\left(t_{j}-s_{j-1}\right)^{2}}{2}\right] \\
& +\left(L_{2} r_{2}+M_{2}\right)\left|\gamma_{m}\right| \sum_{j=1}^{m}\left(\prod_{j}^{m-1}\left|\alpha_{j}\right|\left|\gamma_{j}\right|\right)\left|\beta_{j-1}\right| t_{j}+\left(L_{2} r_{2}+M_{2}\right)\left(s_{m}-t_{m}\right) \\
= & \left(L_{1} r_{2}+M_{1}\right)\left|\gamma_{m}\right| \sum_{j=1}^{m}\left[\left(\prod_{j}^{m-1}\left|\alpha_{j}\right|\left|\gamma_{j}\right|\right) \frac{\left(t_{j}-s_{j-1}\right)^{2}}{2}\right] \\
& +\left(L_{2} r_{2}+M_{2}\right)\left[| \gamma _ { m } | \left\{\sum_{j=1}^{m-1}\left(\prod_{j}^{m-1}\left|\alpha_{j}\right|\right)\left(\prod_{j+1}^{m-1}\left|\gamma_{j+1}\right|\right)\left(s_{j}-t_{j}\right)\right.\right. \\
& \left.\left.+\sum_{j=1}^{m}\left(\prod_{j}^{m-1}\left|\alpha_{j}\right|\left|\gamma_{j}\right|\right)\left|\beta_{j-1}\right| t_{j}\right\}+\left(s_{m}-t_{m}\right)\right]+\left|\gamma_{m} \| \alpha_{0}\right|\left(\prod_{j=1}^{m-1}\left|\alpha_{j}\right|\left|\gamma_{j}\right|\right) \\
\leq & \left.L_{1} r_{2}+M_{1}\right) \Lambda_{3}^{(2)}+\left(L_{2} r_{2}+M_{2}\right) \Lambda_{4}^{(1)}+\Phi_{2} \\
&
\end{aligned}
$$

Since $r^{*}=\max \left\{r_{1}, r_{2}\right\}$, we have $\|\mathcal{A} x\| \leq r^{*}$, which implies $\mathcal{A} B_{r^{*}} \subset B_{r^{*}}$. Next, we will prove that operator $\mathcal{A}$ is a contraction. Let $x, y \in P C^{2,1}(U, \mathbb{R})$. Now, for $t \in\left(s_{i}, t_{i+1}\right] i=$ $0,1,2, \ldots, m$, we get

$$
\begin{aligned}
\mid \mathcal{A} x( & t)-\mathcal{A} y(t) \mid \\
\leq & \sum_{j=1}^{m}\left[\left(\prod_{j}^{m}\left|\alpha_{j}\right|\right)\left(\prod_{j+1}^{m}\left|\gamma_{j+1}\right|\right) \int_{t_{j}}^{s_{j}}|g(r, x(r))-g(r, y(r))| d r\right] \\
& +\sum_{j=1}^{m}\left[\left(\prod_{j}^{m}\left|\alpha_{j}\right|\left|\gamma_{j}\right|\right) \int_{s_{j-1}}^{t_{j}}\left(t_{j}-r\right)|f(r, x(r))-f(r, y(r))| d r\right] \\
& +\sum_{j=1}^{m}\left(\prod_{j}^{m}\left|\alpha_{j}\right|\left|\gamma_{j}\right|\right)\left|\beta_{j-1}\right|\left(\left|g\left(s_{j-1}, x\left(s_{j-1}\right)\right)-g\left(s_{j-1}, y\left(s_{j-1}\right)\right)\right|\right) t_{j} \\
& +\left|\beta_{m}\right|\left(\left|g\left(s_{m}, x\left(s_{m}\right)\right)-g\left(s_{m}, y\left(s_{m}\right)\right)\right|\right) T \\
& +\int_{s_{m}}^{T}(T-r)(|f(r, x(r))-f(r, y(r))|) d r \\
\leq & \left\{\sum_{j=1}^{m}\left[\left(\prod_{j}^{m}\left|\alpha_{j}\right|\right)\left(\prod_{j+1}^{m}\left|\gamma_{j+1}\right|\right) L_{2}\left(s_{j}-t_{j}\right)\right]\right.
\end{aligned}
$$




$$
\begin{aligned}
& +\sum_{j=1}^{m}\left[\left(\prod_{j}^{m}\left|\alpha_{j}\right|\left|\gamma_{j}\right|\right) L_{1}\left(\frac{\left(t_{j}-s_{j-1}\right)^{2}}{2}\right)\right]+\sum_{j=1}^{m}\left(\prod_{j}^{m}\left|\alpha_{j}\right|\left|\gamma_{j}\right|\right)\left|\beta_{j-1}\right| L_{2} t_{j} \\
& \left.+\left|\beta_{m}\right| L_{2} T+L_{1} \frac{\left(T-s_{m}\right)^{2}}{2}\right\}\|x-y\| \\
& \leq\left(\Lambda_{1}^{(2)} L_{1}+\Lambda_{2}^{(1)} L_{2}\right)\|x-y\| .
\end{aligned}
$$

For $t \in\left(t_{i}, s_{i}\right], i=1,2,3, \ldots, m$, we obtain

$$
\begin{aligned}
& |\mathcal{A} x(t)-\mathcal{A} y(t)| \\
& \leq\left|\gamma_{m}\right| \sum_{j=1}^{m-1}\left[\left(\prod_{j}^{m-1}\left|\alpha_{j}\right|\right)\left(\prod_{j+1}^{m-1}\left|\gamma_{j+1}\right|\right) \int_{t_{j}}^{s_{j}}|g(r, x(r))-g(r, y(r))| d r\right] \\
& \quad+\left|\gamma_{m}\right| \sum_{j=1}^{m}\left[\left(\prod_{j}^{m-1}\left|\alpha_{j}\right|\left|\gamma_{j}\right|\right) \int_{s_{j-1}}^{t_{j}}\left(t_{j}-r\right)(|f(r, x(r))-f(r, y(r))|) d r\right] \\
& \quad+\left|\gamma_{m}\right| \sum_{j=1}^{m}\left(\prod_{j}^{m-1}\left|\alpha_{j}\right|\left|\gamma_{j}\right|\right)\left|\beta_{j-1}\right|\left(\left|g\left(s_{j-1}, x\left(s_{j-1}\right)\right)-g\left(s_{j-1}, y\left(s_{j-1}\right)\right)\right|\right) t_{j} \\
& \quad+\int_{s_{m}}^{t_{m}}|g(r, x(r))-g(r, y(r))| d r \\
& \leq\left\{\left|\gamma_{m}\right| \sum_{j=1}^{m}\left[\left(\prod_{j}^{m-1}\left|\alpha_{j}\right|\right)\left(\prod_{j+1}^{m-1}\left|\gamma_{j+1}\right|\right) L_{2}\left(s_{j}-t_{j}\right)+\left(s_{m}-t_{m}\right)\right]\right. \\
& \quad+\left|\gamma_{m}\right| \sum_{j=1}^{m}\left[\left(\prod_{j}^{m-1}\left|\alpha_{j}\right|\left|\gamma_{j}\right|\right) L_{1} \frac{\left(t_{j}-s_{j-1}\right)^{2}}{2}\right] \\
& \left.\quad+\left|\gamma_{m}\right| \sum_{j=1}^{m-1}\left(\prod_{j}^{m-1}\left|\alpha_{j}\right|\left|\gamma_{j}\right|\right)\left|\beta_{j-1}\right| t_{j}\right\}\|x-y\| \\
& \leq \\
& \quad\left(\Lambda_{3}^{(2)} L_{1}+\Lambda_{4}^{(1)} L_{2}\right)\|x-y\| .
\end{aligned}
$$

From the above results, we can conclude that $\|\mathcal{A} x-\mathcal{A} y\| \leq \Omega_{1}\|x-y\|$, which implies that $\mathcal{A}$ is a contraction operator. Therefore, by the Banach contraction mapping principle, operator $\mathcal{A}$ has only one fixed point $x(t)$ in $B_{r^{*}}$ for $t \in U$. Then there exists a unique solution of problem (1.1) such that $\|x\| \leq r^{*}$. This completes the proof.

Example 2.3 Consider the following mixed-order impulsive ordinary differential equations with initial conditions

$$
\left\{\begin{array}{l}
x^{\prime \prime}(t)=\frac{e^{-t^{2}}}{4}\left(\frac{x^{2}(t)+2|x(t)|}{1+|x(t)|}\right)+\frac{3}{2}, \quad t \in(2 i, 2 i+1], i=0,1,2,3, \\
x^{\prime}(t)=\frac{t}{150} \sin |x(t)|+1, \quad t \in(2 i-1,2 i], i=1,2,3, \\
x\left((2 i)^{+}\right)=\left(\frac{i+1}{i+2}\right) x\left((2 i)^{-}\right), \quad x^{\prime}\left((2 i)^{+}\right)=\left(\frac{i+2}{i+3}\right) x^{\prime}\left((2 i)^{-}\right), \\
x\left((2 i-1)^{+}\right)=\left(\frac{i+3}{i+4}\right) x\left((2 i-1)^{-}\right), \quad x(0)=\frac{1}{2}, \quad x^{\prime}(0)=\frac{2}{3} .
\end{array}\right.
$$

Here $s_{i}=2 i, i=0,1,2,3$, and $t_{i}=2 i-1, i=1,2,3, m=3, T=t_{4}=7$. The constants $\alpha_{i}=(i+1) /(i+2), \beta_{i}=(i+2) /(i+3), i=0,1,2,3$, and $\gamma_{i}=(i+3) /(i+4), i=1,2,3$. The in- 
formation in Eq. (2.6) yields by direct computation that $\Lambda_{1}^{(2)} \approx 0.97559, \Lambda_{2}^{(1)} \approx 11.43571$, $\Lambda_{3}^{(2)} \approx 0.83929, \Lambda_{4}^{(1)} \approx 6.83461, \Phi_{1} \approx 0.11429$ and $\Phi_{2} \approx 0.14286$. Setting $f(t, x)=\left(e^{-t^{2}}\left(x^{2}+\right.\right.$ $2|x|)) /(4(1+|x|))+(3 / 2)$ and $g(t, x)=((t \sin |x|) / 150)+1$, we can find Lipschitz constants by

$$
\left|f\left(t_{1}, x\right)-f\left(t_{1}, y\right)\right| \leq \frac{1}{2}|x-y| \quad \text { and } \quad\left|g\left(t_{2}, x\right)-g\left(t_{2}, y\right)\right| \leq \frac{1}{25}|x-y|,
$$

for each $t_{1} \in J, t_{2} \in J^{*}, x, y \in \mathbb{R}$, which satisfy condition $\left(\mathrm{H}_{1}\right)$ as $L_{1}=1 / 2$ and $L_{2}=1 / 25$. Also, we observe that $g(0, \cdot)=1, M_{1}=3 / 2$ and $M_{2}=1$. Then we compute the constants $\Omega_{1}=\max \{0.94522,0.69303\}=0.94522<1$ and $r^{*}=\max \{237.57197,15.80093\}=$ 237.57197. Hence by Theorem 2.2, we obtain that problem (2.6) has a unique solution $x(t)$ on $[0,7]$ such that $\|x\| \leq 237.57197$.

Example 2.4 Let the constants $a, b \in \mathbb{R}, a \neq b$ be given. The function

$$
x(t)= \begin{cases}e^{a t}, & t \in(2 i, 2 i+1], i=0,1,2,3, \ldots, \\ e^{b t}, & t \in(2 i-1,2 i], i=1,2,3, \ldots,\end{cases}
$$

is a unique solution of the problem

$$
\left\{\begin{array}{l}
x^{\prime \prime}(t)=a^{2} x(t), \quad t \in(2 i, 2 i+1], i=0,1,2,3, \ldots, \\
x^{\prime}(t)=b x(t), \quad t \in(2 i-1,2 i], i=1,2,3, \ldots, \\
x\left((2 i)^{+}\right)=e^{2 i(a-b)} x\left((2 i)^{-}\right), \quad x^{\prime}\left((2 i)^{+}\right)=\left(\frac{a}{b}\right) e^{2 i(a-b)} x^{\prime}\left((2 i)^{-}\right), \\
x\left((2 i-1)^{+}\right)=e^{(2 i-1)(b-a)} x\left((2 i-1)^{-}\right), \quad x(0)=1, \quad x^{\prime}(0)=a .
\end{array}\right.
$$

Taking the second and first order derivatives of Eq. (2.7) on $(2 i, 2 i+1], i=0,1,2,3, \ldots$ and $(2 i-1,2 i], i=1,2,3, \ldots$, respectively, the first two equations in Eq. (2.8) hold. It is obvious that $x(0)=1, x^{\prime}(0)=a$. In addition, we get $x\left((2 i)^{+}\right)=e^{2 i a}, x\left((2 i)^{-}\right)=e^{2 i b}, x^{\prime}\left((2 i)^{+}\right)=a e^{2 i a}$, $x^{\prime}\left((2 i)^{-}\right)=b e^{2 i b}, x\left((2 i-1)^{+}\right)=e^{(2 i-1) b}$, and $x\left((2 i-1)^{-}\right)=e^{(2 i-1) a}$. Therefore, function $x(t)$ defined in Eq. (2.7) solves problem (2.8).

\subsection{Mixed-order impulsive fractional differential equations with initial conditions}

The existence results for problem (1.2) which has mixed fractional orders are established in this subsection. In analogy with Theorem 2.1 , the operator $\mathcal{B}: P C^{p, q}(U, \mathbb{R}) \rightarrow$ $P C^{p, q}(U, \mathbb{R})$ is defined by

$$
\mathcal{B} x(t)=\left\{\begin{aligned}
& \alpha_{0}\left(\prod_{j=1}^{i} \alpha_{j} \gamma_{j}\right)+\sum_{j=1}^{i}\left[\left(\prod_{j}^{i} \alpha_{j}\right)\left(\prod_{j+1}^{i} \gamma_{j+1}\right)_{t_{j}} I^{q} g(r, x(r))\left(s_{j}\right)\right] \\
&+\sum_{j=1}^{i}\left[\left(\prod_{j}^{i} \alpha_{j} \gamma_{j}\right)_{s_{j-1}} I^{p} f(r, x(r))\left(t_{j}\right)\right] \\
&+\sum_{j=1}^{i}\left(\prod_{j}^{i} \alpha_{j} \gamma_{j}\right) \beta_{j-1} g\left(s_{j-1}, x\left(s_{j-1}\right)\right) t_{j}+\beta_{i} g\left(s_{i}, x\left(s_{i}\right)\right) t \\
&+s_{i} I^{p} f(r, x(r))(t), \quad t \in J \\
& \alpha_{0} \gamma_{i}\left(\prod_{j=1}^{i-1} \alpha_{j} \gamma_{j}\right)+\gamma_{i} \sum_{j=1}^{i-1}\left[\left(\prod_{j}^{i-1} \alpha_{j}\right)\left(\prod_{j+1}^{i-1} \gamma_{j+1}\right)_{t_{j}} I^{q} g(r, x(r))\left(s_{j}\right)\right] \\
& \quad+\gamma_{i}\left[\sum_{j=1}^{i}\left(\prod_{j}^{i-1} \alpha_{j} \gamma_{j}\right)_{s j-1} I^{p} f(r, x(r))\left(t_{j}\right)\right] \\
& \quad+\gamma_{i} \sum_{j=1}^{i}\left(\prod_{j}^{i-1} \alpha_{j} \gamma_{j}\right) \beta_{j-1} g\left(s_{j-1}, x\left(s_{j-1}\right)\right) t_{j} \\
&+t_{i} I^{q} g(r, x(r))(t), \quad t \in J^{*}
\end{aligned}\right.
$$


where the Riemann-Liouville fractional integral with respect to $r$ of the function of two variables ${ }_{\phi} I^{\theta} h(r, x(r))(\pi), \phi \in\left\{s_{j-1}, t_{j}, s_{i}, t_{i}\right\}, \pi \in\left\{t, s_{j}, t_{j}\right\}, \theta \in\{p, q\}$ and $h \in\{f, g\}$ is defined by Eq. (1.4). By the Banach fixed point theorem, the existence of a unique solution to problem (1.2) can be similarly proved as for Theorem 2.2.

Theorem 2.5 Suppose that functions $f$ and $g$ satisfy condition $\left(\mathrm{H}_{1}\right)$ in Theorem 2.2. If $\Omega_{2}=\max \left\{L_{1} \Lambda_{1}^{(p)}+L_{2} \Lambda_{2}^{(q)}, L_{1} \Lambda_{3}^{(p)}+L_{2} \Lambda_{4}^{(q)}\right\}<1$, then problem (1.2) has a unique solution on $U$.

Next the existence result will be proved by applying the nonlinear alternative for singlevalued maps given as the following statement.

Theorem 2.6 ([35]) Let E be a Banach space, and C be a closed, convex subset of E. Also let $G$ be an open subset of $C$ such that $0 \in G$. Assume that $F: \bar{G} \rightarrow C$ is a continuous, compact (that is, $F(\bar{G})$ is a relatively compact subset of $C$ ) map. Then either

(i) F has a fixed point in $\bar{G}$, or

(ii) There are $a u \in \partial G$ (the boundary of $G$ in $C$ ) and $\lambda \in(0,1)$ with $u=\lambda F(u$ ).

Theorem 2.7 Assume that functions $f: J \times \mathbb{R} \rightarrow \mathbb{R}$ and $g: J^{*} \times \mathbb{R} \rightarrow \mathbb{R}$ with $g(0, \cdot)=1$ are continuous. In addition, we assume that:

$\left(\mathrm{H}_{2}\right)$ There exist continuous nondecreasing functions $\psi_{1}, \psi_{2}:[0, \infty) \rightarrow(0, \infty)$ and continuous functions $w_{1}: J \rightarrow \mathbb{R}^{+}, w_{2}: J^{*} \rightarrow \mathbb{R}^{+}$such that

$$
\left|f\left(t_{1}, x\right)\right| \leq w_{1}\left(t_{1}\right) \psi_{1}(|x|), \quad\left|g\left(t_{2}, x\right)\right| \leq w_{2}\left(t_{2}\right) \psi_{2}(|x|)
$$

for each $\left(t_{1}, x\right) \in J \times \mathbb{R},\left(t_{2}, x\right) \in J^{*} \times \mathbb{R}$.

$\left(\mathrm{H}_{3}\right)$ There exist constants $N_{1}, N_{2}>0$ such that

$$
\frac{N_{1}}{\Phi_{1}+\psi_{1}\left(N_{1}\right)\left\|w_{1}\right\| \Lambda_{1}^{(p)}+\psi_{2}\left(N_{1}\right)\left\|w_{2}\right\| \Lambda_{2}^{(q)}}>1
$$

and

$$
\frac{N_{2}}{\Phi_{2}+\psi_{1}\left(N_{2}\right)\left\|w_{1}\right\| \Lambda_{3}^{(p)}+\psi_{2}\left(N_{2}\right)\left\|w_{2}\right\| \Lambda_{4}^{(q)}}>1 .
$$

Then the mixed-order impulsive fractional differential equations with initial conditions given in Eq. (1.2) have at least one solution on $U$.

Proof Let us, for a positive number $\rho$, define the ball $B_{\rho}=\left\{x \in P C^{p, q}(U, \mathbb{R}):\|x\| \leq \rho\right\}$. It follows that $B_{\rho}$ is a closed, convex subset of $P C^{p, q}(U, \mathbb{R})$. We will show that the operator $\mathcal{B}: P C^{p, q}(U, \mathbb{R}) \rightarrow P C^{p, q}(U, \mathbb{R})$ satisfies all the assumptions of Theorem 2.6. To prove the continuity of $\mathcal{B}$, we define a sequence converging to $x$ by $\left\{x_{n}\right\}$. Then for $t \in J$, we obtain

$$
\begin{aligned}
& \left|\mathcal{B} x_{n}(t)-\mathcal{B} x(t)\right| \\
& \leq \sum_{j=1}^{m}\left[\left(\prod_{j}^{m}\left|\alpha_{j}\right|\right)\left(\prod_{j+1}^{m}\left|\gamma_{j+1}\right|\right){ }_{t_{j}}{ }^{q}\left(\left|g\left(r, x_{n}(r)\right)-g(r, x(r))\right|\right)\left(s_{j}\right)\right]
\end{aligned}
$$




$$
\begin{aligned}
& +\sum_{j=1}^{m}\left[\left(\prod_{j}^{m}\left|\alpha_{j}\right|\left|\gamma_{j}\right|\right) s_{j-1} I^{p}\left(\left|f\left(r, x_{n}(r)\right)-f(r, x(r))\right|\right)\left(t_{j}\right)\right] \\
& +\sum_{j=i}^{m}\left[\left(\prod_{j}^{m}\left|\alpha_{j}\right|\left|\gamma_{j}\right|\right)\left|\beta_{j-1}\right|\left(\left|g\left(s_{j-1}, x_{n}\left(s_{j-1}\right)\right)-g\left(s_{j-1}, x\left(s_{j-1}\right)\right)\right|\right) t_{j}\right] \\
& +\left|\beta_{m}\right|\left(\left|g\left(s_{m}, x_{n}\left(s_{m}\right)\right)-g\left(s_{m}, x\left(s_{m}\right)\right)\right|\right) T \\
& +{ }_{s_{m}} I^{p}\left(\left|f\left(r, x_{n}(r)\right)-f(r, x(r))\right|\right)(T) \rightarrow 0, \quad \text { as } n \rightarrow \infty,
\end{aligned}
$$

and for $t \in J^{*}$, we have

$$
\begin{aligned}
& \left|\mathcal{B} x_{n}(t)-\mathcal{B} x(t)\right| \\
& \leq\left|\gamma_{m}\right| \sum_{j=1}^{m-1}\left[\left(\prod_{j}^{m-1}\left|\alpha_{j}\right|\right)\left(\prod_{j+1}^{m-1}\left|\gamma_{j+1}\right|\right){ }_{t_{j}} I^{q}\left(\left|g\left(r, x_{n}(r)\right)-g(r, x(r))\right|\right)\left(s_{j}\right)\right] \\
& \quad+\left|\gamma_{m}\right| \sum_{j=1}^{m}\left[\left(\prod_{j}^{m-1}\left|\alpha_{j}\right|\left|\gamma_{j}\right|\right) s_{s_{j-1}} I^{p}\left(\left|f\left(r, x_{n}(r)\right)-f(r, x(r))\right|\right)\left(t_{j}\right)\right] \\
& +\left|\gamma_{m}\right| \sum_{j=1}^{m}\left[\left(\prod_{j}^{m-1}\left|\alpha_{j}\right|\left|\gamma_{j}\right|\right)\left|\beta_{j-1}\right|\left(\left|g\left(s_{j-1}, x_{n}\left(s_{j-1}\right)\right)-g\left(s_{j-1}, x\left(s_{j-1}\right)\right)\right|\right) t_{j}\right] \\
& \quad+{ }_{t_{m}} I^{q}\left(\left|g\left(r, x_{n}(r)\right)-g(r, x(r))\right|\right)\left(s_{m}\right) \rightarrow 0, \quad \text { as } n \rightarrow \infty,
\end{aligned}
$$

which implies that operator $\mathcal{B}$ is continuous. Next, we prove the compactness of operator $\mathcal{B}$. Let $x \in B_{\rho}$. Then we have, for $t \in J$,

$$
\begin{aligned}
|\mathcal{B} x(t)| \leq & \left|\alpha_{0}\right|\left(\prod_{j=1}^{m}\left|\alpha_{j}\right|\left|\gamma_{j}\right|\right)+\sum_{j=1}^{m}\left[\left(\prod_{j}^{m}\left|\alpha_{j}\right|\left|\gamma_{j}\right|\right)_{s_{j-1}} I^{p}|f(r, x(r))|\left(t_{j}\right)\right] \\
& \left.+\sum_{j=1}^{m}\left[\left(\prod_{j}^{m}\left|\alpha_{j}\right|\right)\left(\prod_{j+1}^{m}\left|\gamma_{j+1}\right|\right)\right]\right]_{j_{j}}{ }^{q}|g(r, x(r))|\left(s_{j}\right) \\
& +\sum_{j=1}^{m}\left(\prod_{j}^{m}\left|\alpha_{j}\right|\left|\gamma_{j}\right|\right)\left|\beta_{j-1}\right|\left|g\left(s_{j-1}, x\left(s_{j-1}\right)\right)\right| t_{j}+\left|\beta_{m}\right|\left|g\left(s_{m}, x\left(s_{m}\right)\right)\right| T \\
& +{ }_{s_{m}} I^{p}|f(r, x(r))|(T) \\
\leq & \left|\alpha_{0}\right|\left(\prod_{j=1}^{m}\left|\alpha_{j}\right|\left|\gamma_{j}\right|\right)+\left\|w_{1}\right\| \psi_{1}(\rho) \sum_{j=1}^{m}\left[\left(\prod_{j}^{m}\left|\alpha_{j}\right|\left|\gamma_{j}\right|\right) s_{j-1} I^{p}(1)\left(t_{j}\right)\right] \\
& +\left\|w_{2}\right\| \psi_{2}(\rho) \sum_{j=1}^{m}\left[\left(\prod_{j}^{m}\left|\alpha_{j}\right|\right)\left(\prod_{j+1}^{m}\left|\gamma_{j+1}\right|\right) t_{t_{j}} I^{q}(1)\left(s_{j}\right)\right] \\
& +\left\|w_{2}\right\| \psi_{2}(\rho) \sum_{j=1}^{m}\left(\prod_{j}^{m}\left|\alpha_{j}\right|\left|\gamma_{j}\right|\right)\left|\beta_{j-1}\right| t_{j}+\left\|w_{2}\right\| \psi_{2}(\rho)\left|\beta_{m}\right| T \\
& +\left\|w_{1}\right\| \psi_{1}(\rho)_{s_{m}} I^{p}(1)(T) \\
= & \left|\alpha_{0}\right|\left(\prod_{j=1}^{m}\left|\alpha_{j} \| \gamma_{j}\right|\right)
\end{aligned}
$$




$$
\begin{aligned}
& +\left\|w_{1}\right\| \psi_{1}(\rho)\left\{\sum_{j=1}^{m}\left[\left(\prod_{j}^{m}\left|\alpha_{j}\right|\left|\gamma_{j}\right|\right) \frac{\left(t_{j}-s_{j-1}\right)^{p}}{\Gamma(p+1)}\right]+\frac{\left(T-s_{m}\right)^{p}}{\Gamma(p+1)}\right\} \\
& +\left\|w_{2}\right\| \psi_{2}\left(\rho_{2}\right)\left\{\sum_{j=1}^{m}\left[\left(\prod_{j}^{m}\left|\alpha_{j}\right|\right)\left(\prod_{j+1}^{m}\left|\gamma_{j+1}\right|\right) \frac{\left(s_{j}-t_{j}\right)^{q}}{\Gamma(q+1)}\right]\right. \\
& \left.+\sum_{j=1}^{m}\left(\prod_{j}^{m}\left|\alpha_{j}\right|\left|\gamma_{j}\right|\right)\left|\beta_{j-1}\right| t_{j}+\left(\left|\beta_{m}\right| T\right)\right\} \\
& =\Phi_{1}+\left\|w_{1}\right\| \psi_{1}(\rho) \Lambda_{1}^{(p)}+\left\|w_{2}\right\| \psi_{2}\left(\rho_{2}\right) \Lambda_{2}^{(q)}:=K_{1},
\end{aligned}
$$

and for $t \in J^{*}$,

$$
\begin{aligned}
& |\mathcal{B} x(t)| \leq\left|\alpha_{0}\right|\left|\gamma_{m}\right|\left(\prod_{j=1}^{m-1}\left|\alpha_{j}\right|\left|\gamma_{j}\right|\right)+\left|\gamma_{m}\right| \sum_{j=1}^{m}\left[\left(\prod_{j}^{m-1}\left|\alpha_{j}\right|\left|\gamma_{j}\right|\right) s_{j-1} I^{p}|f(r, x(r))|\left(t_{j}\right)\right] \\
& +\left|\gamma_{m}\right| \sum_{j=1}^{m-1}\left[\left(\prod_{j}^{m-1}\left|\alpha_{j}\right|\right)\left(\prod_{j+1}^{m-1}\left|\gamma_{j+1}\right|\right)\right]{ }_{t j} I^{q}|g(r, x(r))|\left(s_{j}\right) \\
& +\left|\gamma_{m}\right| \sum_{j=1}^{m}\left(\prod_{j}^{m-1}\left|\alpha_{j}\right|\left|\gamma_{j}\right|\right)\left|\beta_{j-1}\right|\left|g\left(s_{j-1}, x\left(s_{j-1}\right)\right)\right| t_{j} \\
& +{ }_{t_{m}} I^{q}|g(r, x(r))|\left(s_{m}\right) \\
& \leq\left|\alpha_{0}\right|\left|\gamma_{m}\right|\left(\prod_{j=1}^{m-1}\left|\alpha_{j}\right|\left|\gamma_{j}\right|\right)+\left\|w_{1}\right\| \psi_{1}(\rho)\left|\gamma_{m}\right| \sum_{j=1}^{m}\left[\left(\prod_{j}^{m-1}\left|\alpha_{j}\right|\left|\gamma_{j}\right|\right) s_{j-1} I^{p}(1)\left(t_{j}\right)\right] \\
& +\left\|w_{2}\right\| \psi_{2}(\rho)\left|\gamma_{m}\right| \sum_{j=1}^{m-1}\left[\left(\prod_{j}^{m-1}\left|\alpha_{j}\right|\right)\left(\prod_{j+1}^{m-1}\left|\gamma_{j+1}\right|\right)\right]{ }_{t_{j}} I^{q}(1)\left(s_{j}\right) \\
& +\left\|w_{2}\right\| \psi_{2}(\rho)\left|\gamma_{m}\right| \sum_{j=1}^{m}\left(\prod_{j}^{m-1}\left|\alpha_{j}\right|\left|\gamma_{j}\right|\right)\left|\beta_{j-1}\right| t_{j} \\
& +\left\|w_{2}\right\| \psi_{2}(\rho)_{t_{m}} I^{q}(1)\left(s_{m}\right) \\
& =\left|\alpha_{0}\right|\left|\gamma_{m}\right|\left(\prod_{j=1}^{m-1}\left|\alpha_{j}\right|\left|\gamma_{j}\right|\right) \\
& +\left\|w_{1}\right\| \psi_{1}(\rho)\left\{\left|\gamma_{m}\right| \sum_{j=1}^{m}\left[\left(\prod_{j}^{m-1}\left|\alpha_{j}\right|\left|\gamma_{j}\right|\right) \frac{\left(t_{j}-s_{j-1}\right)^{p}}{\Gamma(p+1)}\right]\right\} \\
& +\left\|w_{2}\right\| \psi_{2}(\rho)\left\{\left|\gamma_{m}\right| \sum_{j=1}^{m-1}\left[\left(\prod_{j}^{m-1}\left|\alpha_{j}\right|\right)\left(\prod_{j+1}^{m-1}\left|\gamma_{j+1}\right|\right) \frac{\left(s_{j}-t_{j}\right)^{q}}{\Gamma(q+1)}\right]\right. \\
& \left.+\left|\gamma_{m}\right| \sum_{j=1}^{m}\left[\left(\prod_{j}^{m-1}\left|\alpha_{j}\right|\left|\gamma_{j}\right|\right)\left|\beta_{j-1}\right| t_{j}\right]+\frac{\left(s_{m}-t_{m}\right)^{q}}{\Gamma(q+1)}\right\} \\
& =\Phi_{2}+\left\|w_{1}\right\| \psi_{1}(\rho) \Lambda_{3}^{(p)}+\left\|w_{2}\right\| \psi_{2}(\rho) \Lambda_{4}^{(q)}:=K_{2} .
\end{aligned}
$$

Setting $K=\max \left\{K_{1}, K_{2}\right\}$, we obtain $\|\mathcal{B} x\| \leq K$, which implies that $\mathcal{B} B_{\rho}$ is a uniformly bounded set. Next we will prove that the set $\mathcal{B} B_{\rho}$ is equicontinuous. Let $\tau_{1}, \tau_{2}$ be two 
points in $U$ such that $\tau_{1}<\tau_{2}$ and let $x \in B_{\rho}$. Then, for $\tau_{1}, \tau_{2} \in J$, we have

$$
\begin{aligned}
\left|\mathcal{B} x\left(\tau_{2}\right)-\mathcal{B} x\left(\tau_{1}\right)\right| & =\left|\beta_{i} g\left(s_{i}, x\left(s_{i}\right)\right)\left(\tau_{2}-\tau_{1}\right)+{ }_{s_{i}} I^{p} f(r, x(r))\left(\tau_{2}\right)-{ }_{s_{i}} I^{p} f(r, x(r))\left(\tau_{1}\right)\right| \\
& \leq\left|\beta_{i}\right| \psi_{2}(\rho)\left\|w_{2}\right\|\left(\tau_{2}-\tau_{1}\right)+\frac{\psi_{1}(\rho)\left\|w_{1}\right\|}{\Gamma(p+1)}\left|\left(\tau_{2}-s_{i}\right)^{p}-\left(\tau_{1}-s_{i}\right)^{p}\right| \\
& \rightarrow 0 \quad \text { as } \tau_{1} \rightarrow \tau_{2}, i=0,1, \ldots, m,
\end{aligned}
$$

and, for $\tau_{1}, \tau_{2} \in J^{*}$,

$$
\begin{aligned}
\left|\mathcal{B} x\left(\tau_{2}\right)-\mathcal{B} x\left(\tau_{1}\right)\right| & =\left|t_{i} I^{q} g(r, x(r))\left(\tau_{2}\right)-{ }_{t_{i}} I^{q} g(r, x(r))\left(\tau_{1}\right)\right| \\
& \leq\left\|w_{2}\right\| \psi_{2}(\rho) \frac{1}{\Gamma(q+1)}\left|\left(\tau_{2}-t_{i}\right)^{q}-\left(\tau_{1}-t_{i}\right)^{q}\right| \\
& \rightarrow 0 \quad \text { as } \tau_{1} \rightarrow \tau_{2}, i=1,2, \ldots, m .
\end{aligned}
$$

From the above results we deduce that $\mathcal{B} B_{\rho}$ is an equicontinuous set. Hence the set $\mathcal{B} B_{\rho}$ is relatively compact. Applying the Arzelá-Ascoli theorem, the operator $\mathcal{B}$ is completely continuous.

Finally, we will show that condition (ii) of Theorem 2.6 is not true. Let $x$ be a solution of problem (1.2). Now, we consider the operator equation $x=\lambda \mathcal{B} x$ for $\lambda \in(0,1)$. From the method to compute $K_{1}, K_{2}$, we have, for $t \in J$,

$$
\|x\| \leq \Phi_{1}+\psi_{1}(\|x\|)\left\|w_{1}\right\| \Lambda_{1}^{(p)}+\psi_{2}(\|x\|)\left\|w_{2}\right\| \Lambda_{2}^{(q)},
$$

and, for $t \in J^{*}$,

$$
\|x\| \leq \Phi_{2}+\psi_{1}(\|x\|)\left\|w_{1}\right\| \Lambda_{3}^{(p)}+\psi_{2}(\|x\|)\left\|w_{2}\right\| \Lambda_{4}^{(q)} .
$$

Then we get

$$
\frac{\|x\|}{\Phi_{1}+\psi_{1}(\|x\|)\left\|w_{1}\right\| \Lambda_{1}^{(p)}+\psi_{2}(\|x\|)\left\|w_{2}\right\| \Lambda_{2}^{(q)}} \leq 1
$$

and

$$
\frac{\|x\|}{\Phi_{2}+\psi_{1}(\|x\|)\left\|w_{1}\right\| \Lambda_{3}^{(p)}+\psi_{2}(\|x\|)\left\|w_{2}\right\| \Lambda_{4}^{(q)}} \leq 1
$$

By assumption $\left(\mathrm{H}_{3}\right)$, there exist two positive constants $N_{1}$ and $N_{2}$ such that $\|x\| \neq N_{1}, N_{2}$. Let $N=\min \left\{N_{1}, N_{2}\right\}$ and define $V=\left\{x \in B_{\rho}:\|x\|<N\right\}$. It is obvious that $\mathcal{B}: \bar{V} \rightarrow$ $P C^{p, q}(U, \mathbb{R})$ is continuous and completely continuous. Hence, there is no $x \in \partial V$ such that $x=\lambda \mathcal{B} x$ for some $\lambda \in(0,1)$. Therefore, the result follows from Theorem 2.6(i), so that $\mathcal{B}$ has a fixed point $x \in \bar{V}$ which is a solution of problem (1.2) on $U$. The proof is completed.

Theorem 2.7 is a very general result because the functions $f$ and $g$ are bounded by nonlinear functions. However, we can get some special cases of linear results as following corollaries. 
Corollary 2.8 Suppose that functions $f: J \times \mathbb{R} \rightarrow \mathbb{R}$ and $g: J^{*} \times \mathbb{R} \rightarrow \mathbb{R}$ with $g(0, \cdot)=1$ satisfy the condition:

$\left(\mathrm{H}_{4}\right)\left|f\left(t_{1}, x\right)\right| \leq Q_{1}|x|+Q_{2}$ and $\left|g\left(t_{2}, x\right)\right| \leq Q_{3}|x|+Q_{4}$, where $Q_{1}, Q_{3} \geq 0$ and $Q_{2}, Q_{4}>0$, for each $\left(t_{1}, x\right) \in J \times \mathbb{R},\left(t_{2}, x\right) \in J^{*} \times \mathbb{R}$.

If $Q_{1} \Lambda_{1}^{(p)}+Q_{3} \Lambda_{2}^{(q)}<1$ and $Q_{1} \Lambda_{3}^{(p)}+Q_{3} \Lambda_{4}^{(q)}<1$, then the impulsive fractional differential problem (1.2) has at least one solution on $U$.

Corollary 2.9 Suppose that condition $\left(\mathrm{H}_{2}\right)$ in Theorem 2.7 holds with $\psi_{1}(\cdot)=\psi_{2}(\cdot) \equiv 1$, that is,

$$
\left|f\left(t_{1}, x\right)\right| \leq w_{1}\left(t_{1}\right), \quad\left|g\left(t_{2}, x\right)\right| \leq w_{2}\left(t_{2}\right), \quad \text { for each }\left(t_{1}, x\right) \in J \times \mathbb{R},\left(t_{2}, x\right) \in J^{*} \times \mathbb{R} .
$$

Then the impulsive problem (1.2) has at least one solution on $U$.

Example 2.10 Consider the following mixed-order impulsive fractional differential equations with initial conditions

$$
\left\{\begin{array}{l}
{ }_{u} D^{\frac{3}{2}} x(t)=f(t, x(t)), \quad t \in(2 i, 2 i+1], i=0,1,2,3, \\
{ }_{v} D^{\frac{1}{2}} x(t)=g(t, x(t)), \quad t \in(2 i-1,2 i], i=1,2,3, \\
x\left((2 i)^{+}\right)=\left(\frac{i+1}{i+2}\right) x\left((2 i)^{-}\right), \quad x^{\prime}\left((2 i)^{+}\right)=\left(\frac{i+2}{i+3}\right) x^{\prime}\left((2 i)^{-}\right), \\
x\left((2 i-1)^{+}\right)=\left(\frac{i+3}{i+4}\right) x\left((2 i-1)^{-}\right), \quad x(0)=\frac{1}{2}, \quad x^{\prime}(0)=\frac{2}{3} .
\end{array}\right.
$$

Here the constants $s_{i}, \alpha_{i}, \beta_{i}, i=0,1,2,3$, and $t_{i}, \gamma_{i}, i=1,2,3, m=3, T=7$ are defined as in Example 2.3 , including constants $\Phi_{1} \approx 0.11428, \Phi_{2} \approx 0.14285$. In addition, we put $p=3 / 2$ and $q=1 / 2$, which can be computed so that $\Lambda_{1}^{(3 / 2)} \approx 1.60117, \Lambda_{2}^{(1 / 2)} \approx 11.23742$, $\Lambda_{3}^{(3 / 2)} \approx 1.26271$ and $\Lambda_{4}^{(1 / 2)} \approx 6.47929$.

$$
\text { (i) } f(t, x)=\frac{6 x^{2}+10|x|}{5(5+6|x|)}+\frac{e^{-t^{2}}}{3+t} \text { and } g(t, x)=\frac{t^{2}}{36(4+t)^{2}} \tan ^{-1}|x|+\frac{t}{\sqrt{t+1}}+1 \text {. }
$$

Then we see that the Lipschitz condition $\left(\mathrm{H}_{1}\right)$ holds due to

$$
\left|f\left(t_{1}, x\right)-f\left(t_{1}, y\right)\right| \leq \frac{2}{5}|x-y|, \quad\left|g\left(t_{2}, x\right)-g\left(t_{2}, y\right)\right| \leq \frac{1}{100}|x-y|,
$$

for $t_{1} \in J, t_{2} \in J^{*}$ and $x, y \in \mathbb{R}$, with $L_{1}=2 / 5$ and $L_{2}=1 / 100$. Also, we get $g(0, \cdot)=1$. Then $\Omega_{2}=\max \{0.75284,0.56988\}=0.75284<1$. Theorem 2.5 now implies that the impulsive mixed-order fractional differential problem (2.9) with functions in (i) has a unique solution on $[0,7]$.

(ii) $f(t, x)=\frac{1}{t+5}\left(\frac{x^{8}}{3\left(x^{6}+1\right)}+\frac{1}{2}\right)$ and $g(t, x)=\frac{t}{15}\left(\frac{|x|^{9}}{4\left(|x|^{7}+1\right)}+1\right)+e^{-15 t}$.

Observe that $g(0, \cdot)=1$. Since function $g$ is defined on $(2 i-1,2 i], i=1,2,3$, and $e^{-15 t}<t / 15$ for $t>0.26826$, we have the following estimates:

$$
\left|f\left(t_{1}, x\right)\right| \leq \frac{1}{t+5}\left(\frac{1}{3} x^{2}+\frac{1}{2}\right), \quad\left|g\left(t_{2}, x\right)\right| \leq \frac{t}{15}\left(\frac{1}{4} x^{2}+2\right),
$$


for $t_{1} \in J, t_{2} \in J^{*}$ and $x \in \mathbb{R}$. Choosing $w_{1}(t)=1 /(t+5), w_{2}=t / 15, \psi_{1}(x)=(1 / 3) x^{2}+(1 / 2)$, and $\psi_{2}(x)=(1 / 4) x^{2}+2$, we get $\left\|w_{1}\right\|=1 / 5,\left\|w_{2}\right\|=2 / 5$. Furthermore, we find that there exist two constants $N_{1}, N_{2}$ such that $N_{1} \in(1.29286,4.70714)$ and $N_{2} \in(1.25216,3.11147)$ satisfying condition $\left(\mathrm{H}_{3}\right)$ of Theorem 2.7. Then the conclusion follows from Theorem 2.7, namely, the mixed fractional order impulsive problem (2.9) with functions in (ii) has at least one solution on $[0,7]$.

$$
\text { (iii) } f(t, x)=\frac{x^{8}}{10\left(|x|^{7}+1\right)}+\frac{3}{2} \cos ^{2} t \text { and } g(t, x)=\frac{7|x|^{9} \sin ^{2} t}{100\left(x^{8}+1\right)}+\frac{t^{2}}{t^{2}+1}+1 \text {. }
$$

Here we observe that

$$
|f(t, x)| \leq \frac{1}{10}|x|+\frac{3}{2}, \quad|g(t, x)| \leq \frac{7}{100}|x|+\frac{73}{37},
$$

with $Q_{1}=1 / 10, Q_{2}=3 / 2, Q_{3}=7 / 100, Q_{4}=73 / 37$ and $g(0, \cdot)=1$. Therefore, we get $Q_{1} \Lambda_{1}^{(3 / 2)}+Q_{3} \Lambda_{2}^{(1 / 2)} \approx 0.94674<1$ and $Q_{1} \Lambda_{3}^{(3 / 2)}+Q_{3} \Lambda_{4}^{(1 / 2)} \approx 0.59874<1$. Hence, from Corollary 2.8, problem (2.9) with functions in (iii) has at least one solution on $[0,7]$.

\section{Acknowledgements}

Not applicable.

\section{Funding}

This research was funded by King Mongkut's University of Technology North Bangkok. Contract no. KMUTNB-60-ART-068.

\section{Availability of data and materials}

Data sharing not applicable to this article as no data sets were generated or analyzed during the current study.

\section{Competing interests}

The authors declare that they have no competing interests.

Authors' contributions

All authors contributed equally to this work. All authors read and approved the final manuscript.

\section{Author details}

1 Intelligent and Nonlinear Dynamic Innovations Research Center, Department of Mathematics, Faculty of Applied Science, King Mongkut's University of Technology North Bangkok, Bangkok, Thailand. ${ }^{2}$ Department of Mathematics, University of loannina, loannina, Greece. ${ }^{3}$ Nonlinear Analysis and Applied Mathematics (NAAM)—Research Group, Department of Mathematics, Faculty of Science, King Abdulaziz University, Jeddah, Saudi Arabia.

\section{Publisher's Note}

Springer Nature remains neutral with regard to jurisdictional claims in published maps and institutional affiliations.

Received: 14 February 2019 Accepted: 3 June 2019 Published online: 13 June 2019

\section{References}

1. Samko, S.G., Kilbas, A.A., Marichev, O.I.: Fractional Integrals and Derivatives: Theory and Applications. Gordon \& Breach, Yverdon (1993)

2. Podlubny, I.: Fractional Differential Equations. Academic Press, San Diego (1999)

3. Kilbas, A.A., Srivastava, H.M., Trujillo, J.J.: Theory and Applications of Fractional Differential Equations. North-Holland Mathematics Studies, vol. 204. Elsevier, Amsterdam (2006)

4. Baleanu, D., Diethelm, K., Scalas, E., Trujillo, J.J.: Fractional Calculus Models and Numerical Methods. Series on Complexity, Nonlinearity and Chaos. World Scientific, Boston (2012)

5. Agarwal, R.P., Zhou, Y., He, Y.: Existence of fractional neutral functional differential equations. Comput. Math. Appl. 59, 1095-1100 (2010)

6. Baleanu, D., Mustafa, O.G., Agarwal, R.P.: On $L^{p}$-solutions for a class of sequential fractional differential equations. Appl. Math. Comput. 218, 2074-2081 (2011)

7. Ahmad, B., Nieto, J.J.: Riemann-Liouville fractional integro-differential equations with fractional nonlocal integral boundary conditions. Bound. Value Probl. 2011, Article ID 36 (2011)

8. Ahmad, B., Ntouyas, S.K., Alsaedi, A.: New existence results for nonlinear fractional differential equations with three-point integral boundary conditions. Adv. Differ. Equ. 2011, Article ID 107384 (2011) 
9. O'Regan, D., Stanek, S.: Fractional boundary value problems with singularities in space variables. Nonlinear Dyn. 71, 641-652 (2013)

10. Ahmad, B., Ntouyas, S.K., Alsaedi, A.: A study of nonlinear fractional differential equations of arbitrary order with Riemann-Liouville type multistrip boundary conditions. Math. Probl. Eng. 2013, Article ID 320415 (2013)

11. Ahmad, B., Nieto, J.J.: Boundary value problems for a class of sequential integrodifferential equations of fractional order. J. Funct. Spaces Appl. 2013, Article ID 149659 (2013)

12. Liu, X., Jia, M., Ge, W.: Multiple solutions of a p-Laplacian model involving a fractional derivative. Adv. Differ. Equ. 2013, Article ID 126 (2013)

13. da Vanterler, J., Sousa, C., Capelas de Oliveira, E.: On the $\psi$-Hilfer fractional derivative. Commun. Nonlinear Sci. Numer. Simul. 60, 72-91 (2018)

14. da Vanterler, J., Sousa, C., Capelas de Oliveira, E.: On the $\Psi$-fractional integral and applications. Comput. Appl. Math. 38, 4 (2019). https://doi.org/10.1007/s40314-019-0774-z

15. Samoilenko, A.M., Perestyuk, N.A.: Impulsive Differential Equations. World Scientific, Singapore (1995)

16. Lakshmikantham, V., Bainov, D.D., Simeonov, P.S.: Theory of Impulsive Differential Equations. World Scientific, Singapore (1989)

17. Benchohra, M., Henderson, J., Ntouyas, S.K.: Impulsive Differential Equations and Inclusions. Hindawi Publishing Corporation, New York (2006)

18. Yukunthorn, W., Ntouyas, S.K., Tariboon, J.: Impulsive multiorders Riemann-Liouville fractional differential equations. Discrete Dyn. Nat. Soc. 2015, Article ID 603893 (2015)

19. Thaiprayoon, C., Tariboon, J., Ntouyas, S.K.: Impulsive fractional boundary-value problems with fractional integral jump conditions. Bound. Value Probl. 2014, Article ID 17 (2014)

20. Yukunthorn, W., Ahmad, B., Ntouyas, S.K., Tariboon, J.: On Caputo-Hadamard type fractional impulsive hybrid systems with nonlinear fractional integral conditions. Nonlinear Anal. Hybrid Syst. 19, 77-92 (2016)

21. Ahmad, B., Wang, G.: Impulsive anti-periodic boundary value problem for nonlinear differential equations of fractional order. Comput. Math. Appl. 59, 1341-1349 (2010)

22. Feckan, M., Zhou, Y., Wang, J.R.: On the concept and existence of solution for impulsive fractional differential equations. Commun. Nonlinear Sci. Numer. Simul. 17, 3050-3060 (2012)

23. Ke, T.D., Lan, D.: Decay integral solutions for a class of impulsive fractional differential equations in Banach spaces, Fract. Calc. Appl. Anal. 17(1), 96-121 (2014)

24. Wang, J.R., Feckan, M., Zhou, Y.: Relaxed controls for nonlinear fractional impulsive evolution equations. J. Optim. Theory Appl. 156, 13-32 (2013)

25. Hernndez, E., O'Regan, D.: On a new class of abstract impulsive differential equations. Proc. Am. Math. Soc. 141, 1641-1649 (2013)

26. Suganya, S., Baleanu, D., Kalamani, P., Arjunan, M.M.: On fractional neutral integro-differential systems with state-dependent delay and non-instantaneous impulses. Adv. Differ. Equ. 2015, Article ID 372 (2015)

27. Agarwal, R.P., Hristova, S., O'Regan, D.: Noninstantaneous impulses in Caputo fractional differential equations and practical stability via Lyapunov functions. J. Franklin Inst. 354, 3097-3119 (2017)

28. Agarwal, R., O'Regan, D., Hristova, S.: Monotone iterative technique for the initial value problem for differential equations with noninstantaneous impulses. Appl. Math. Comput. 298, 45-56 (2017)

29. Feckan, M., Wang, J.R., Zhou, Y.: Periodic solutions for nonlinear evolution equations with non-instantaneous impulses. Nonauton. Dyn. Syst. 1, 93-101 (2014)

30. da Vanterler, J., Sousa, C., dos Santos Oliveira, D., Capelas de Oliveira, E.: On the existence and stability for noninstantaneous impulsive fractional integrodifferential equation. Math. Methods Appl. Sci. 42, 1249-1261 (2019)

31. Yang, D., Wang, J., O'Regan, D.: On the orbital Hausdorff dependence of differential equations with non-instantaneous impulses. C. R. Acad. Sci. Paris, Ser. I 356, 150-171 (2018)

32. Agarwal, R.P., Hristova, S., O'Regan, D.: Non-Instantaneous Impulses in Differential Equations. Springer, Cham (2017)

33. Wang, J.R., Feckan, M.: Non-instantaneous Impulsive Differential Equations: Basic Theory and Computation. IOP Publishing, Bristol (2018)

34. Muslim, M., Kumar, A., Feckan, M.: Existence, uniqueness and stability of solutions to second order nonlinear differential equations with non-instantaneous impulses. J. King Saud Univ., Sci. 30, 204-213 (2018)

35. Granas, A., Dugundji, J.: Fixed Point Theory. Springer, New York (2003)

\section{Submit your manuscript to a SpringerOpen ${ }^{\circ}$ journal and benefit from:}

- Convenient online submission

- Rigorous peer review

- Open access: articles freely available online

- High visibility within the field

Retaining the copyright to your article

Submit your next manuscript at $\gg$ springeropen.com 